\title{
Acute inhibition of estradiol synthesis impacts vestibulo-ocular reflex adaptation and cerebellar long-term potentiation in male rats
}

\author{
Cristina V. Dieni ${ }^{1} \cdot$ Aldo Ferraresi $^{1} \cdot$ Jacqueline A. Sullivan $^{2} \cdot$ Sivarosa Grassi $^{1}$ \\ Vito E. Pettorossi ${ }^{1} \cdot$ Roberto Panichi $^{1}$ (D)
}

Received: 13 March 2017/Accepted: 6 September 2017/Published online: 23 September 2017

(C) The Author(s) 2017. This article is an open access publication

\begin{abstract}
The vestibulo-ocular reflex (VOR) adaptation is an ideal model for investigating how the neurosteroid 17 beta-estradiol (E2) contributes to the modification of behavior by regulating synaptic activities. We hypothesized that E2 impacts VOR adaptation by affecting cerebellar synaptic plasticity at the parallel fiber-Purkinje cell (PF) synapse. To verify this hypothesis, we investigated the acute effect of blocking E2 synthesis on gain increases and decreases in adaptation of the VOR in male rats using an oral dose $(2.5 \mathrm{mg} / \mathrm{kg})$ of the aromatase inhibitor letrozole. We also assessed the effect of letrozole on synaptic plasticity at the PF synapse in vitro, using cerebellar slices from male rats. We found that letrozole acutely impaired both gain increases and decreases adaptation of the VOR without altering basal ocular-motor performance. Moreover, letrozole prevented long-term potentiation at the PF synapse (PF-LTP) without affecting long-term depression (PF-LTD). Thus, in male rats neurosteroid E2 has a relevant impact on VOR adaptation and affects exclusively PF-LTP. These findings suggest that E2 might regulate changes in VOR adaptation by acting locally on cerebellar and extra-cerebellar synaptic plasticity sites.
\end{abstract}

Keywords Neurosteroids · Plasticity · Vestibulo-ocular reflex adaptation $\cdot$ Cerebellum $\cdot$ Purkinje cell

Roberto Panichi

roberto.panichi@unipg.it

1 Department of Experimental Medicine, Section of Physiology and Biochemistry, University of Perugia, 06127 Perugia, Italy

2 Department of Philosophy, Western University, London, ON N6A5B8, Canada

\section{Introduction}

Neural networks rely on multiple mechanisms to make the diverse forms of plasticity that underlie adaptive behavior and learning possible. Mounting evidence suggests that neurosteroid 17 beta-estradiol (E2) might regulate behavioral processes by influencing spine density, synaptogenesis and long-term potentiation (LTP) (Foy et al. 1999; Woolley and McEwen 1994; Xu and Zhang 2006).

According to the classical view, E2 acts via slow genomic mechanisms to produce delayed effects on cellular activity and behavior (Farr et al. 1995; McEwen and Alves 1999). However, it is also well known that E2 may rapidly influence neuronal activity through fast nongenomic mechanisms involving specific E2 membrane receptors (ERs: ER $\alpha$, ER $\beta$ and GPER) (Morissette et al. 2008; Mukai et al. 2007, 2010; Raz et al. 2008; Woolley 2007).

In addition to gonadal origin, E2 is locally synthesized in the central nervous system from cholesterol (Baulieu 1997; Compagnone and Mellon 2000), by an aromatasedependent conversion of testosterone (Kimoto et al. 2001). Local E2 is a region-specific neurosteroid and can play an important role in modulating neuronal activity due to its ability to rapidly reach higher concentrations than the gonadal hormone (Hojo et al. 2009; Mukai et al. 2010).

The modulatory role of neurosteroid E2 on synaptic plasticity has been demonstrated in vestibular nuclei, basal ganglia and hippocampus by acute as well as long-lasting inhibition of the E2 pathway, which impairs LTP induction (Grassi et al. 2009a, b, 2010, 2012, 2013; Pettorossi et al. 2013; Scarduzio et al. 2013; Vierk et al. 2012, 2014). Consistent with these findings, it has been shown that acute inhibition of E2 synthesis also exerts rapid effects on spatial learning, working memory and fear extinction (Alejandre-Gomez et al. 2007; Graham and Milad 2014; Moradpour et al. 2006). 
These discoveries indicate that neurosteroid E2 may rapidly influence learning at the behavioral and synaptic levels. Nevertheless, the physiological significance of this rapid action on synaptic activities and its consequences for behavior has yet to be clarified.

A model well suited to this purpose is adaptive re-calibration of the vestibulo-ocular reflex (VOR), a cerebellardependent form of motor learning induced by visuovestibular stimuli (Boyden and Raymond 2003; Ito et al. 1974; Lisberger and Miles 1980).

The VOR circuitry is mediated by a direct pathway through the vestibular nuclei and a more complex inhibitory loop in the cerebellar cortex that includes the flocculus and paraflocculus.

Although multiple plastic mechanisms might be distributed between cerebellar and vestibular sites to calibrate the VOR, the primary mechanism responsible for encoding gain increase and decrease adaptation are thought to be LTP at the parallel fiber-Purkinje cell synapses (PF-LTP) and long-term depression (PF-LTD) that occurs at the same or a different subset of synapses, respectively (Boyden et al. 2004; Boyden and Raymond 2003; Broussard et al. 2011; Coesmans et al. 2004; Hansel et al. 2001).

So far, evidence indicates that the cerebellum expresses estrogen receptors ( $\alpha, \beta$, GPER) and can produce key enzymes for E2 formation (Hazell et al. 2009; Hedges et al. 2012; Sakamoto et al. 2003; Tsutsui et al. 2011). Moreover, an effect of chronic E2 administration in ovariectomized mice on Purkinje cell (PC) plasticity and on VOR adaptation has been shown (Andreescu et al. 2007).

Thus, we hypothesized that neurosteroid E2 impacts the expression of VOR adaptation and regulates cerebellar synaptic plasticity.

To test this hypothesis, we acutely blocked E2 synthesis using systemic administration of letrozole (LTZ), an imidazole derivative inhibitor of the aromatase enzyme, and evaluated the effect on gain increases and decreases adaptation of the VOR in male rats. In addition, we investigated the acute effect of LTZ on PF-LTP and PFLTD in male rat cerebellar slices.

Our findings contribute to delineating the physiological significance of local E2 synthesis in the brain and provide new insights about the function of the neurosteroid E2 on memory formation.

\section{Materials and methods}

\section{Ethics statement}

All experiments labeled in this paper were performed in accordance with protocols approved by the Ethical Committees of the University of Perugia, in compliance with the guidelines of the Italian Ministry of Health, national laws on animal research (Legislative Decree 116/92) and The European Communities Council directive on animal research (N. 86/609/EEC). All efforts were made to minimize the number of animals used and their suffering.

\section{Subjects}

The experiments were performed on male Wistar rats ( $n=36$, Harlan, Italy) to avoid any possible influences of estrogenic fluctuation during estrous cycle on both electrophysiological recordings and behavioral tests (Pettorossi et al. 2011). Animals were maintained on a $12 \mathrm{~h}$ light-dark cycle with food and water available ad libitum. Twenty rats (P35-P60, weighting 140-290 g) were employed in behavioral tests and 16 (P28-P49, weighting 90-190 g) in electrophysiological recordings.

\section{Behavioral}

\section{Surgical preparation and eye recording}

To restrain the head of the rats during the tests, a small dental acrylic socket was implanted surgically in the skull under general anesthesia using ketamine $(50 \mathrm{mg} / \mathrm{kg})$ and medetomidine $(0.5 \mathrm{mg} / \mathrm{kg})$.

We used an infrared light projection technique (Fig. 1b) to measure right eye movements (Barmack and Nelson 1987). Before each recording, we anesthetized the right eye with $0.4 \%$ oxybuprocaine and glued (Histoacryl ${ }^{\circledR}$, Aesculap AG, Tuttlingen, Germany) a small silicone rubber cylinder (diameter $1 \mathrm{~mm}$, height $3 \mathrm{~mm}$ ) bearing an infrared light-emitting diode (IrLED) aligned with the visual axis. The narrow beam of infrared light was detected by a photosensitive $\mathrm{X}-\mathrm{Y}$ position detector (SC-50, United Detector Technology, Hawthorne, CA, USA), fixed relative to the head. The circular photosensitive $\mathrm{X}-\mathrm{Y}$ position detector had a $50 \mathrm{~mm}$ diameter and gave a continuous $\mathrm{X}-\mathrm{Y}$ voltage proportional to the position of the incident centroid of infrared light. The eye movement recording system was calibrated by moving the IrLED using a model mimicking rat eye through a known angular displacement. The system had a sensitivity of $0.2 \mathrm{~min}$ of arc and was linear for eye deviations of $\pm 15^{\circ}$ within $4 \%$ and for eye deviations of $\pm 30^{\circ}$ within $7 \%$. Data were digitized with a sampling frequency of $500 \mathrm{~Hz}$.

\section{Equipment, stimuli and drug administration}

During each behavioral experiment, the animal body was restrained on a servo-controlled turntable and the head was immobilized at the center of the rotation (Fig. 1a). All rats were placed with the head tilted $35^{\circ}$ nose down to position 
A

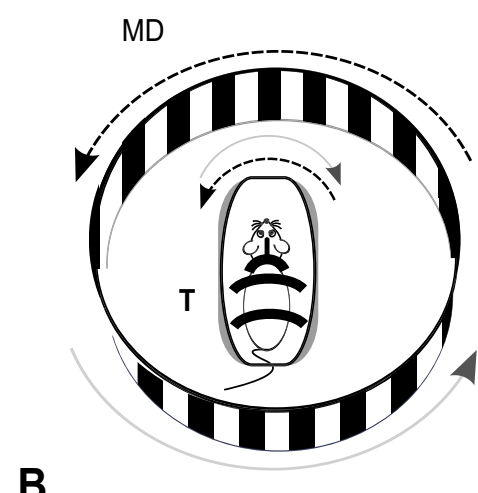

B

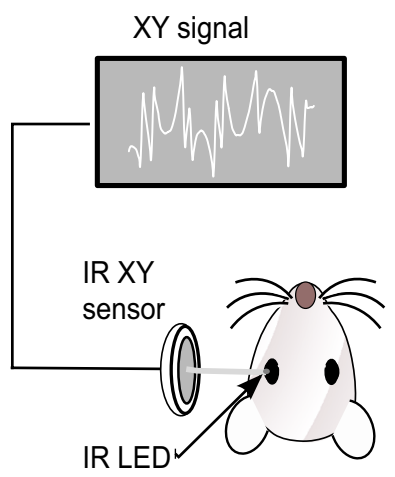

C

Gain increase
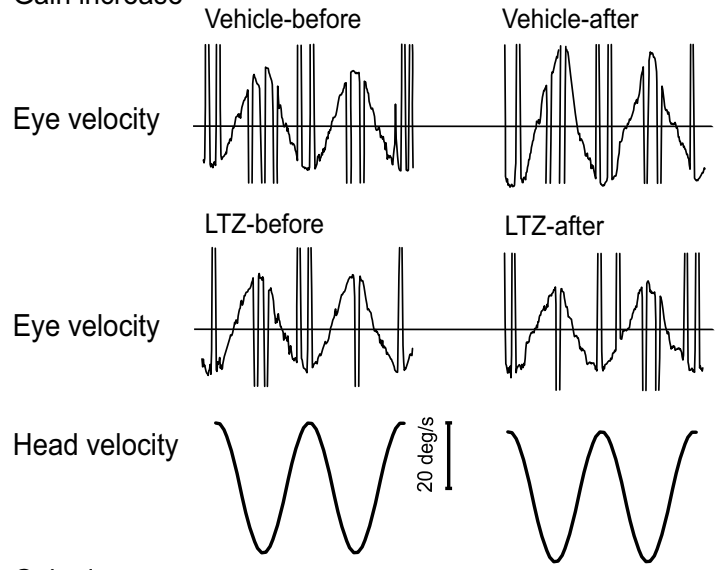

Gain decrease

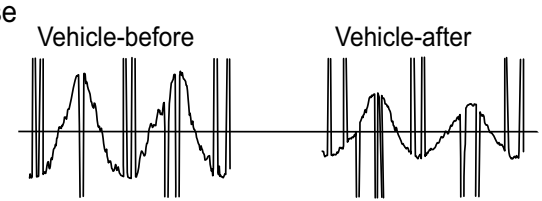

Eye velocity

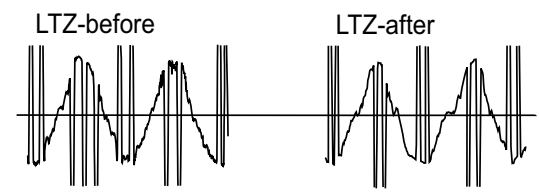

Fig. 1 VOR in vehicle- and LTZ-treated rats. a Cartoon showing the experimental setup. Rats were blocked with the head fixed at the rotation center of a turntable $(\mathrm{T})$ positioned inside a striped moving drum (MD). The VOR was evoked in the dark by horizontal sinusoidal oscillations of the turntable while the OKR was evoked by drum rotations in light. Adaptive changes of the VOR were induced by paring the turntable rotation with the rotation of the striped drum at identical peak velocity and at conditioning frequency of $0.2 \mathrm{~Hz}$ for $45 \mathrm{~min}$. In gain-down conditioning, the turntable and the drum were sinusoidally rotated in the same direction (black dashed arrows), whereas in the gain up the drum was moved in the opposite direction

the lateral semicircular canals approximately earth horizontally. We used sinusoidal oscillation on the vertical axis (yaw stimulation) to apply vestibular stimuli. Optokinetic experiments were performed by placing the animal, immobilized on the turntable, inside a servo-controlled optokinetic drum (diameter $1.15 \mathrm{~m}$; height $1.20 \mathrm{~m}$ ) (Fig. 1a). The drum interior wall was painted with vertical black and white stripes (width $6 \mathrm{~cm}$; angle $6^{\circ}$ ). The drum rotated on the vertical axis and was backlit by two 9-W LED lamps placed $50 \mathrm{~cm}$ outside the drum. A black disk was placed under the rat to prevent it from seeing the turntable and floor.

The VOR was evoked by trials of turntable oscillation in the dark (peak-to-peak amplitude of $30^{\circ}$ and frequencies $0.05,0.1,0.2$ and $0.4 \mathrm{~Hz}$ ), which were composed of four cycles of sinusoidal rotation. The OKR was evoked by delivering $5 \mathrm{~min}$ of constant velocity $(5-30 \mathrm{deg} / \mathrm{s})$ from the turntable (gray solid arrows). b Eye movement recording technique. On the rat cornea was attached a small plastic stem bearing an infrared light-emitting diode (LED). The LED projected a narrow beam of infrared light (IR) onto a dual axis infrared $\mathrm{X}-\mathrm{Y}$ photodiode (sensor) that encoded the horizontal and vertical eye position. c Representative VOR and turntable velocity traces recorded in the dark at $0.2 \mathrm{~Hz}$ before (left) and after (right) gain conditioning in vehicle and letrozole (LTZ) treated rats for gain increase (top) and decrease (bottom) adaptation. LTZ treatment induced a decrease rather than an increase in gain-up conditioning and prevented gain decrease in gain-down conditioning

illuminated drum rotation in the horizontal plane and temporo-nasal direction.

For the VOR adaptation the conditioning stimuli consisted of turntable and drum sinusoidal oscillations on the vertical axis in the light (Boyden et al. 2006; Kimpo et al. 2005). Two different sets of oscillation parameters were used to induce VOR decrease (gain-down conditioning) and increase (gain-up conditioning), respectively (Fig. 1a). The VOR gain decrease was induced by presenting in phase sinusoidal vestibular and optokinetic stimuli for 45 min (gain-down conditioning stimulus: the turntable and the drum moved exactly in the same direction with identical peak velocity of $20 \mathrm{deg} / \mathrm{s}$ and amplitude of $30^{\circ}$ at frequency of $0.2 \mathrm{~Hz}$ ). The VOR gain increase was induced by presenting $180^{\circ}$ out-of-phase sinusoidal vestibular and optokinetic stimuli for $45 \mathrm{~min}$ (gain-up conditioning stimulus: the turntable and the drum moved in the opposite direction with the same peak velocity of $20 \mathrm{deg} / \mathrm{s}$ at 
frequency of $0.2 \mathrm{~Hz}$ ). During the conditioning period, we presented an attention-normalizing stimulus every $60 \mathrm{~s}$ (usually a sharp noise like a clap).

Before the recordings, awake rats were fed with an oral dose of vehicle ( $0.1 \mathrm{~g}$ of wet cereals) or LTZ $(2.5 \mathrm{mg} / \mathrm{kg}$ of body weight). Oral doses of LTZ were freshly prepared by mixing wet cereal with the drug.

\section{Protocol}

Behavioral testing began when recovery was complete, usually 1 week after surgery (van Alphen et al. 2001). A few days before performing the first conditioning session the rats were acclimatized to the head restraint for 30-min trials and the visuo-motor ability was evaluated by estimating the VOR and OKR. VOR adaptation was studied in 16 rats divided into two groups (8 animals for each group) that were tested in two separate conditioning sessions. In each group, four animals were alternatively treated with the oral dose of vehicle in the first session and the oral dose of LTZ in the second session, whereas the other four rats underwent the opposite sequence of treatment. The sessions were separated by 8 days because our preliminary data (three rats) indicated that in animals that were allowed to be freely moving during the normal light-dark cycle the VOR returned to normal 3 days after the conditioning (Boyden and Raymond 2003; Miles and Eighmy 1980). LTZ or vehicle was given $2.5 \mathrm{~h}$ before starting the recordings. At the beginning of each session the basal VOR and OKR were determined, animals were then trained with the conditioning stimulus (gain-up conditioning for one group and gain-down for the other one) and at the end of each session the VOR was again measured. Typical HVOR traces pre- and post-conditioning were observed for both LTZ and vehicle treatment (Fig. 1c).

In addition, basal VOR and OKR were evaluated in four other rats at different time points (160, 190, $210 \mathrm{~min})$ after a single oral dose of LTZ.

\section{Electrophysiology}

\section{Slice preparation}

Rats were anesthetized and perfused intracardially with ice-cold modified artificial CSF containing the following (in $\mathrm{mM}$ ): 110 choline chloride, 26 D-glucose, $2.5 \mathrm{MgCl}_{2}$, $2.5 \mathrm{KCl}, 1.25 \mathrm{Na}_{2} \mathrm{PO}_{4}, 0.5 \mathrm{CaCl}_{2}, 1.3 \mathrm{Na}$-ascorbate, $3 \mathrm{Na}-$ pyruvate, and $25 \mathrm{NaHCO}_{3}$, bubbled with $95 \% \mathrm{O}_{2} / 5 \% \mathrm{CO}_{2}$. The brain was removed and 300- $\mu \mathrm{m}$-thick sagittal slices of the cerebellar vermis were prepared using a vibratome (Leica VT1200, Leica Instruments). Slices were incubated at $37{ }^{\circ} \mathrm{C}$ for $30 \mathrm{~min}$ in recording solution containing the following (in mM): $125 \mathrm{NaCl}, 2.5 \mathrm{KCl}, 1.25 \mathrm{Na}_{2} \mathrm{PO}_{4}, 2$
$\mathrm{CaCl}_{2}, 1 \mathrm{MgCl}_{2}, 25 \mathrm{NaHCO}_{3}$, and 25 D-glucose bubbled with $95 \% \mathrm{O}_{2} / 5 \% \mathrm{CO}_{2}$, and then transferred to room temperature in the same solution.

Patch pipettes were filled with the following $(\mathrm{mM})$ : $150 \mathrm{~K}$-gluconate, $1 \mathrm{MgCl}_{2}, 1.1$ EGTA, 5 HEPES and 10 phosphocreatine, $\mathrm{pH} 7.2$ and $300 \mathrm{mOsm}$.

\section{Whole cell patch clamp recordings and drugs}

Synaptic responses from Purkinje cells (PCs) were evoked using one patch pipette filled with extracellular solution placed in the molecular layer to stimulate the PFs (duration $100 \mu \mathrm{s}$; intensity $10-50 \mu \mathrm{A}$ ). Paired pulse facilitation (PPF) was assessed by applying two pulses with an interval of $50 \mathrm{~ms}$ at the PFs.

All the recordings were performed in the presence of gabazine (SR95531) $10 \mu \mathrm{M}$.

Pairing stimulations of $\mathrm{PF}-\mathrm{CF}$ were performed placing a theta glass pipette filled with ACSF near the PC layer to stimulate the CF (intensity $1-20 \mu \mathrm{A}$, duration $100 \mu \mathrm{s}$ ). The pipette was repositioned, and the stimulus intensity was adjusted until the voltage required to elicit an all-or-none response was minimized to eliminate $\mathrm{PF}$ activation.

Tetanization was delivered in current-clamp mode for $5 \mathrm{~min}$ at $1 \mathrm{~Hz}$ stimulating PFs either alone (LTP protocol) or in combination with CFs (LTD protocol). All recordings were made at a holding potential of $-60 \mathrm{mV}$ to prevent spontaneous neuronal discharge. Series and input resistances $\left(R_{\mathrm{S}}\right.$ and $\left.R_{\text {in }}\right)$ were monitored throughout the experiments by applying hyperpolarizing voltage steps $(-10 \mathrm{mV})$ at the beginning of each sweep. Recordings were excluded if $R_{\mathrm{S}}$ or $R_{\text {in }}$ varied by $>20 \%$ over the course of the experiments.

Voltages were not corrected for junction potentials and currents were filtered at $2 \mathrm{kHz}$ and sampled at $10 \mathrm{kHz}$ (MultiClamp 700A; Molecular Devices).

We used letrozole (LTZ) to block E2 synthesis. Stock solution of LTZ $(10 \mathrm{mM})$ was dissolved in dimethyl sulphoxide (DMSO) and diluted to the final concentration (LTZ $100 \mathrm{nM}$, DMSO <0.01\%) in recording solution.

Drugs and chemicals were obtained from SigmaAldrich, Tocris Bioscience, or Ascent Scientific.

\section{Statistical evaluations}

All data were expressed as mean $\pm \mathrm{SD}$. To minimize type I error, we set the alpha level at 0.05 and accepted significant results with $p<0.05$ for all statistical tests. The sample size was estimated prior to achieving an analysis power of 0.80 or higher in post hoc evaluation (Gpower). Normality was assayed using the Shapiro-Wilk test. All data sets satisfied normality criteria and two-tailed Student's $t$ test or ANOVA designs were used to evaluate differences among 
two or multiple samples, respectively (Statistica, StatSoft and OriginPro, Origin Lab Corporation). Post hoc analyses, if required for multiple comparisons, were made by Tukey's tests. The homogeneity of the variance between populations was verified by Levene's test.

Fittings were made by single exponential and sinusoidal functions for electrophysiological and behavioral evaluations, respectively, with the goodness of the fit estimated by calculating the Chi-square (OriginPro software). The best fit was obtained by minimizing the mean square error between the data and the curve (Levenberg-Marquardt algorithm).

\section{Behavioral evaluation}

Eye and table or drum position traces were analyzed using OriginPro software. For the VOR evaluation, eye position signals (four response cycles for each delivered trial) were differentiated and any quick phase was removed (LabView, National Instruments, Austin, TX, USA). The differentiated traces were fitted with a sine wave and the ratio between fitted peak velocity and table peak velocity was used to calculate the VOR gain at each frequency tested. The VOR phase was assessed by the difference between eye fitted velocity trace phases and table velocity phases, considering zero phase when fitted eye peak velocity appeared at the same time but in the opposite direction with respect to the peak table velocity. Phase lead was indicated as positive and phase lag as negative.

The OKR gain was calculated by dividing eye peak velocity by drum peak velocity.

Traces containing anomalous eye movements or motion artifacts were excluded from the analysis.

We evaluated with two-way repeated measures ANOVA the effect of LTZ (comparing LTZ and vehicle) on gain and phase across frequencies and velocities for the basal VOR and OKR, respectively. Moreover, two-way repeated measures ANOVA was employed to evaluate the effect of LTZ at different time points after LTZ treatment (160, 190, $210 \mathrm{~min}$ ) on gain and phase across frequencies and velocities for the VOR and OKR, respectively. A three-way repeated measures ANOVA was used to analyze the influence of the conditioning training (comparing responses before and after conditioning) and LTZ (comparing LTZ and vehicle) on VOR gain and phase across the frequencies. The $F$ values expressed the significant differences concerning the main factors (LTZ $\times$ vehicle, time, conditioning training, frequency and velocities) and their interaction.

\section{Electrophysiological evaluation}

Recordings were acquired by pClamp10 (Molecular Devices) and evaluated using Axograph X (AxoGraph Software) and OriginPro software.

EPSC rise time (20-80\%) was calculated from EPSC onset. The decay time constant was measured by best fitting the single exponential to the EPSC decay. The average of EPSC amplitudes recorded at the beginning ( $t=5-10 \mathrm{~min})$ of each experiment was considered as baseline. The influence of LTZ on rise time, decay time and $R_{\text {in }}$ was evaluated comparing baseline ( $t=5-10 \mathrm{~min})$ and $\mathrm{LTZ}(t=50-55 \mathrm{~min})$ by a two-tailed paired $t$ test and on the EPSC amplitudes comparing baseline ( $t=5-10 \mathrm{~min})$ and LTZ at two different time points ( $t=15-20 \mathrm{~min}$ and $t=50-55 \mathrm{~min}$ ) by a one-way repeated measures ANOVA. Changes in the EPSC amplitude induced by LTD and LTP protocols were expressed as a percentage of the baseline. Long-term potentiation and depression were established when the EPSC amplitude, measured at the interval between 25 and $30 \mathrm{~min}$ after tetanization ( $t=40-45 \mathrm{~min}$ ), was significantly increased or decreased compared to baseline (two-tailed paired $t$ test). LTZ influence on LTP and LTD frequency occurrence was statistically assessed by the Fisher's exact test. The effect of LTZ on LTP and LTD induction was evaluated comparing the EPSC amplitude before $(t=5-10 \mathrm{~min})$ and after tetanization ( $t=40-45 \mathrm{~min}$ ) in control and in LTZ by a twoway repeated measures ANOVA.

The $F$ value indicated a significant difference concerning the main factors (LTZ $\times$ control, induction of longterm plasticity) and their interaction.

\section{Results}

\section{VOR adaptation requires neurosteroid E2 synthesis}

To verify whether neurosteroid E2 can influence the VOR adaptation, we acutely blocked the synthesis of E2 with LTZ in 16 male rats and investigated the effect on the induction of VOR adaptive changes.

Before the recordings, we treated the animals with an oral dose of vehicle in one experimental session and with an oral dose of LTZ $(2.5 \mathrm{mg} / \mathrm{kg})$ in a second session performed 8 days later. Alternatively, we used a reverse treatment, treating the animals first with LTZ and 8 days after with vehicle. At the beginning of each recording session we tested the basal VOR and OKR. Thereafter, the animals were trained with gain-up or gain-down conditioning (eight rats for each conditioning training) and at the end of each session we again recorded the VOR. 
Acute E2 synthesis inhibition does not affect basal VOR and $O K R$

We evaluated the VOR gain and phase by horizontally rotating the head at several sinusoidal oscillation frequencies $(0.05,0.1,0.2,0.4 \mathrm{~Hz})$ and the OKR using a striped rotating drum at velocities of 1, 5, 15, $30 \mathrm{deg} / \mathrm{s}$ (Figs. 1, 2). In vehicle rats, the VOR gain at $0.05 \mathrm{~Hz}$ was $0.36 \pm 0.07$ and progressively increased reaching $0.78 \pm 0.1$ at $0.4 \mathrm{~Hz}$ ( $n=16$, Fig. 2a). Yet, the VOR phase, at $0.05 \mathrm{~Hz}$ was $56^{\circ} \pm 13^{\circ}$ and decreased to a value close to zero at $0.4 \mathrm{~Hz}$ ( $n=16$, Fig. $2 \mathrm{a}$ ). The OKR gain (monocularly analyzed during temporo-nasal stimuli) at $1 \mathrm{deg} / \mathrm{s}$ was $0.59 \pm 0.07$ and decreased to $0.31 \pm 0.08$ at $30 \mathrm{deg} / \mathrm{s}$ ( $n=16$ Fig. $2 \mathrm{~b}$ ). The general dynamic characteristics of VOR and OKR were in line with previous findings in the literature (Collewijn et al. 1980; Sirkin et al. 1985).

No significant differences were found comparing the VOR gain and phase in vehicle- and LTZ-treated rats (Fig. 2a; gain: $F_{\text {LTZ } \times \text { vehicle }}(1,14)=0.016, p=0.899$; $F_{\text {frequency }}(3,42)=114.72, p<0.001, F_{\text {interaction }}(3$, $42)=8.713, p<0.001$; phase: $F_{\text {LTZ } \times \text { vehicle }}(1,14)=1.01$, $p=0.32, F_{\text {frequency }}(3,42)=1399.64, p<0.001, F_{\text {inter- }}$ action $(3,42)=315, p<0.001$, two-way repeated measures ANOVA). Similarly, no LTZ effect was detected on the OKR analysis (Fig. 2b; gain: $F_{\text {LTZ } \times \text { vehicle }}(1,14)=0.119$, $p=0.732 ; F_{\text {velocity }}(3,42)=366.1, p<0.001 ; F_{\text {interaction }}$ $(3,42)=301, p<0.001$, two-way repeated measures ANOVA).

Moreover, to exclude LTZ influences on basal reflexes over time, we tested the VOR and OKR in four additional rats at different time points (160, 190, $210 \mathrm{~min})$ after LTZ administration. No changes were found for either reflex across the observed timeframe (VOR gain: $F_{\text {time }(160 \times 190 \times 210)}(2,9)=0.67, p=0.53, F_{\text {frequency }}(3$, $27)=8.7, p<0.01, F_{\text {interaction }}(3,27)=0.3, p=0.88$; OKN gain: $F_{\text {time }(160 \times 190 \times 210)}(2,9)=0.75, p=0.42$, $F_{\text {velocity }}(3,27)=5.6, p<0.05, F_{\text {interaction }}(3,27)=0.41$, $p=0.83$; two-way repeated measures ANOVA).

Thus, neither the VOR nor OKR were affected by acute E2 depletion, indicating that neurosteroid E2 was not required for basal reflexes.

\section{E2 synthesis inhibition acutely impairs gain decrease and increase adaptation of the VOR}

To adaptively modify the VOR, we paired the horizontal rotations of the head with a striped optokinetic drum rotation. Moving sinusoidally the head and the drum in the same direction with identical velocity (gain-down conditioning) caused an adaptive decrease in VOR gain, whereas moving the drum in the opposite direction (gain-up conditioning) caused an increase in VOR gain (Figs. 1, 2, 3).

Effect of acute E2 synthesis inhibition by LTZ on adaptive decrease in VOR gain The influence of $\mathrm{LTZ}$ on gain decrease adaptation of the VOR was investigated in eight randomly designated rats. The VOR gain and phase were assessed at several frequencies $(0.05,0.1,0.2$ and $0.4 \mathrm{~Hz})$ before and after $45 \mathrm{~min}$ of conditioning at $0.2 \mathrm{~Hz}$, in vehicle- and LTZ-treated animals (Figs. 1, 2, 3a, b).

The overall analysis of the gain showed a significant difference between pre- and post-conditioning, between LTZ and vehicle-treated animals and between frequencies
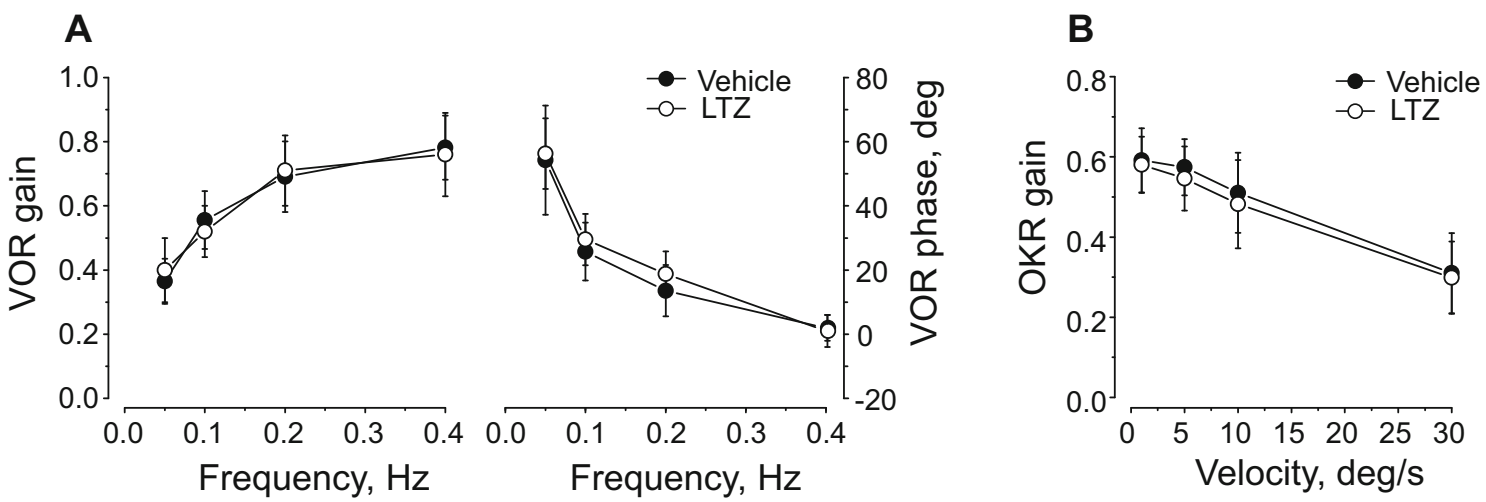

Fig. 2 Acute E2 synthesis inhibition by LTZ does not affect basal ocular-motor reflexes. a VOR gain (left) and phase (right) measured in the dark at several head rotation frequencies $(0.05,1,0.2,0.4 \mathrm{~Hz})$ in vehicle- and LTZ-treated rats. b OKR gain measured in the light at several drum velocity rotations $(1,5,10,30 \mathrm{deg} / \mathrm{s})$ in vehicle and
LTZ. Gain and phase showed typical changes across the frequencies or velocity for the VOR and the OKR, respectively. No significant differences were found comparing reflexes after vehicle and LTZ treatments ( $p>0.05$, two-way repeated measures ANOVA). Values are mean $\pm \mathrm{SD}$ of measurements in 16 rats 
Fig. 3 LTZ affects VOR adaptation. a In vehicle-treated rats, gain-up (eight rats) and gaindown (eight rats) conditioning at $0.2 \mathrm{~Hz}$ induced adaptive VOR gain increase and decrease, respectively, while phase changes were significant only after gainup conditioning. Comparison between changes in VOR gain and phase induced by gain-down (b) and gain-up (c) conditioning in LTZ and vehicle rats. b In LTZ rats conditioning prevented gain decrease compared to vehicle (three-way repeated measures ANOVA). No differences were detected on phase. In c, in LTZ rats conditioning induced gain decrease instead of an increase and a reduction in phase delay compared to vehicle (three-way repeated measures ANOVA). In $\mathbf{b}, \mathbf{c}$, the gain is expressed in percentage (increase or decrease) as post-conditioning change respect pre-conditioning values normalized to 0 (dotted line). The phase is expressed as difference between post-conditioning and pre-conditioning values normalized to 0 (dotted line). Each rat was used either in gainup or gain-down conditioning.

Values are mean $\pm \mathrm{SD}$. Asterisk indicates a significant variation in VOR gain and phase $\left({ }^{*} p<0.05\right.$, $* * p<0.01$, *** $p<0.005$; post hoc analysis by Tukey's tests)

[Figs. 1, 2, 3a, b; gain: $F_{\text {LTZ } \times \text { vehicle }}(1,28)=6.2, p<0.05$; $F_{\text {conditioning training }}(1,28)=18.2, p<0.001 ; F_{\text {frequencies }}(3$, $74)=540.3, p<0.001$; three-way repeated measures ANOVA]. In LTZ-treated animals, the conditioning did not induce significant modification in VOR gain (Figs. 1, 2, 3b; gain before and after conditioning, $p=0.39$; post hoc Tukey's tests) while in animals treated with vehicle the conditioning caused a robust gain decrease, on average, by $36 \pm 8.6 \%$ (Figs. 1, 2, 3a, b; gain before and after conditioning, $p<0.001$; post hoc Tukey's tests). Conversely, the VOR phase was not influenced by conditioning or by LTZ, while significant changes were still detected across the frequencies [Fig. 3a, b; $F_{\text {LTZ } \times \text { vehicle }}(1,28)=0.83$, $p=0.37: F_{\text {conditioning training }}(1,28)=0.21, p=0.64$; $F_{\text {frequencies }}(3,74)=7.3, p<0.05$; three-way repeated measures ANOVA].
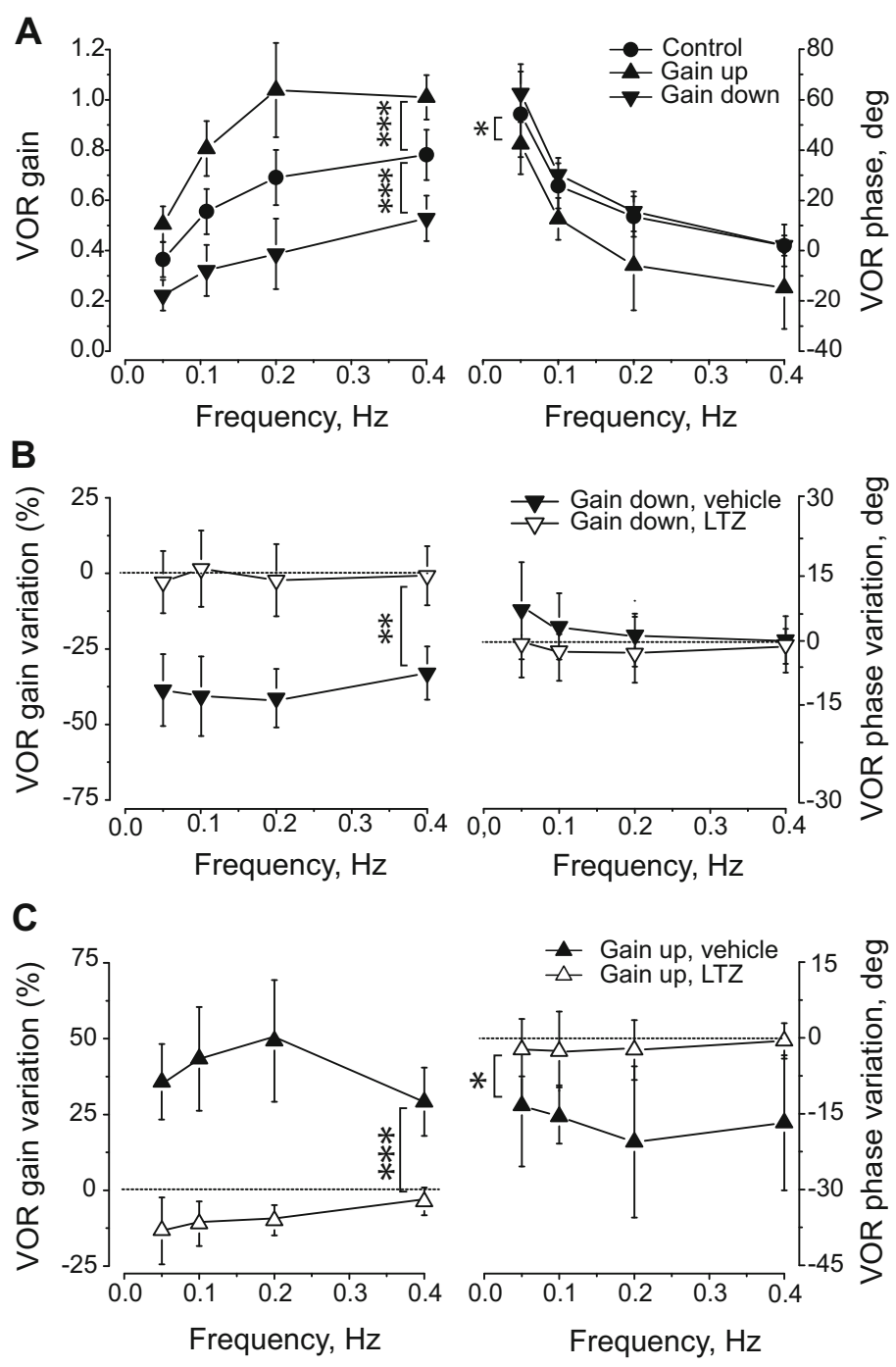

Thus, the conditioning did not induce significant change in VOR gain after LTZ treatment.

Effect of acute E2 synthesis inhibition by LTZ on adaptive increase in VOR gain In another group of male rats $(n=8)$, we studied the influence of LTZ on gain increase adaptation of the VOR assessing the gain and phase as we previously did for the gain decrease. The LTZ influence on the gain adaptation was largely significant compared to the vehicle (Figs. 1, 2, 3c). Also significant were the differences in gain between pre- and post-conditioning and between frequencies [Fig. 3a, c; gain: $F_{\text {LTZ } \times \text { vehicle }}(1$, $28)=34.39, p<0.001 ; F_{\text {conditioning training }}(1,28)=4.6$, $p<0.05 ; F_{\text {frequencies }}(3,74)=214.7, p<0.001 ; F_{\text {LTZ } \times \text { ve- }}$ hicle $\times$ frequencies $(3.74)=13.18, p=0.001$; three-way repeated measures ANOVA]. The LTZ influence was so 
Table 1 VOR gain assessed in the first (Ist) and second (IInd) experimental session before and after conditioning in male rats alternately treated with vehicle and LTZ

\begin{tabular}{|c|c|c|c|c|c|}
\hline \multirow[t]{3}{*}{ Conditioning } & \multirow[t]{3}{*}{ Treatment } & \multicolumn{4}{|l|}{ VOR gain } \\
\hline & & \multicolumn{2}{|l|}{ Ist } & \multicolumn{2}{|l|}{ IInd } \\
\hline & & Before & After & Before & After \\
\hline \multirow[t]{2}{*}{ Gain-up conditioning } & Vehicle & $0.68 \pm 0.12^{+}$ & $1.05 \pm 0.15^{\#}$ & $0.67 \pm 0.14^{+}$ & $1.01 \pm 0.23^{\#}$ \\
\hline & LTZ & $0.72 \pm 0.13^{+}$ & $0.63 \pm 0.07^{\dagger}$ & $0.71 \pm 0.11^{+}$ & $0.67 \pm 0.09^{\dagger}$ \\
\hline \multirow[t]{2}{*}{ Gain-down conditioning } & Vehicle & $0.70 \pm 0.10^{+}$ & $0.42 \pm 0.14^{\#}$ & $0.72 \pm 0.11^{\dagger}$ & $0.44 \pm 0.15^{\#}$ \\
\hline & LTZ & $0.69 \pm 0.10^{+}$ & $0.67 \pm 0.09^{\dagger}$ & $0.70 \pm 0.12^{+}$ & $0.68 \pm 0.13^{\dagger}$ \\
\hline
\end{tabular}

The reported gain was obtained at $0.2 \mathrm{~Hz}$. Values are mean \pm SD from eight male rats for gain-up conditioning and eight male rats for gain down. Significant differences in the gain were found between LTZ and vehicle and before and after conditioning while no differences were detected between the Ist and the IInd session [gain increase: $F_{\mathrm{LTZ} \times \text { Vehicle }}(1,12)=14.6, p<0.005 ; F_{\text {conditioning }}(1,12)=10.1, p<0.01$; $F_{\text {Ist } \times \text { IInd session }}(1,12)=0.07, p=0.8$; gain down: $F_{\text {LTZ } \times \text { vehicle }}(1,12)=12.5, p<0.005 ; F_{\text {conditioning }}(1$, $12)=19.9, p<0.001 ; F_{\text {Ist } \times \text { Ind session }}(1,12)=0.08, p=0.78$; repeated measures ANOVA]. The achieved power of the analysis was 0.95

+ No significant difference in the gain measured before conditioning while

$\#, \uparrow$ No significant difference in the gain measured after conditioning in the Ist and in the IInd session $(p>0.8$, post hoc Tukey's tests) remarkably notable that the gain-up conditioning induced a gain decrease rather than an increase (Figs. 1, 2, 3c; gain before and after conditioning, $p<0.05$; post hoc Tukey's tests) and the largest variation was observed at the frequency of $0.2 \mathrm{~Hz}$. On the other hand, in vehicle animals the conditioning caused a significant and large gain increase, on average, by $43 \pm 9.3 \%$ (Figs. 1, 2, 3a, c; gain before and after conditioning, $p<0.001$; post hoc Tukey's tests) reaching a maximum at the frequency of $0.2 \mathrm{~Hz}$.

Moreover, we also found that the VOR phase was significantly influenced by LTZ, conditioning training and frequencies [Fig. 3a, c; $F_{\text {LTZ } \times \text { vehicle }}(1,28)=8.3, p<0.01$; $F_{\text {conditioning training }}(1,28)=7.7, p<0.01 ; F_{\text {frequencies }}(3$, $74)=12.5, p<0.005$; three-way repeated measures ANOVA]. Specifically, we found that, in LTZ the change of VOR phase was precluded (Fig. 3c; phase before and after conditioning, $p=0.96$; post hoc Tukey's tests) whereas in vehicle the phase was significantly delayed (Fig. 3c; phase before and after conditioning, $p<0.005$; post hoc Tukey's tests).

In summary, in gain increase adaptation LTZ converted the increase into decrease and prevented VOR phase changes.

\section{The protocol does not affect VOR adaptive changes}

No significant influences were found on basal and VOR gain-adaptive changes when we compared the gain values in the first and second sessions (Table 1). These results indicated that the sequence used to alternately treat the animals by vehicle and LTZ in two sessions separated by 8 days did not influence the results and no bias was added by our protocol.

\section{E2 synthesis is necessary for PF-LTP but not for PF- LTD}

To assess whether the neurosteroid E2 impacts rapid regulation of cerebellar synaptic plasticity, we blocked E2 synthesis by perfusing LTZ in male rat acute cerebellar slices and investigated the effect on LTP and LTD induction at the parallel fiber to Purkinje cell synapse (PF-LTP and PF-LTD).

\section{E2 synthesis inhibition does not affect intrinsic and synaptic properties of PCs}

First, we determined whether LTZ per se, could affect the intrinsic and synaptic properties of PCs by evaluating input resistance $\left(R_{\mathrm{in}}\right)$, rise time, decay time and the excitatory post-synaptic current (EPSC) amplitude evoked by focal stimulation of PF. The intrinsic and synaptic properties were measured at different time points during control and LTZ ( $n=6)$. No significant differences were found in the rise time, decay time and $R_{\text {in }}$ comparing control with LTZ (Table 2; control period was measured at $t=5-10 \mathrm{~min}$ and LTZ at $t=50-55 \mathrm{~min} ; p=0.81, p=0.80, p=0.65$, respectively, two-tailed $t$ test), as well as in the EPSC amplitudes [Table 2; control period was measured at $t=5$ $10 \mathrm{~min}$ and LTZ at $t=15-20 \mathrm{~min}$ and $t=50-55 \mathrm{~min} ; F(2$, $118)=0.78, p=0.37$, one-way repeated measures ANOVA]. Additionally, to verify whether LTZ affected the release probability $(\mathrm{Pr})$ we compared the paired pulse ratio (PPR) before and after LTZ and no significant changes were detected (Table 2; $p=0.74$, two-tailed $t$ test). Thus, the presence of LTZ per se in the bath did not modify the passive and synaptic properties of PCs. 
Table 2 Basal synaptic propriety of cerebellar Purkinje cells from male rats

\begin{tabular}{llllll}
\hline Condition & PPR & EPSC amplitude $(\mathrm{pA})$ & Rise time $(20-80 \%, \mathrm{~ms})$ & Decay time constant $(\mathrm{ms})$ & $R_{\text {in }}(\mathrm{M} \Omega)$ \\
\hline Control & $1.04 \pm 0.185$ & $254.4 \pm 14.3$ & $2.02 \pm 0.43$ & $18.73 \pm 2.41$ & $164.7 \pm 46.2$ \\
Letrozole & $1.03 \pm 0.169$ & $247.6 \pm 12.4$ & $1.97 \pm 0.39$ & $19.39 \pm 1.95$ & $156.3 \pm 44.8$ \\
\hline
\end{tabular}

Values are mean \pm SD from cerebellar slices of six rats

E2 synthesis inhibition prevents PF-LTP without altering $P F-L T D$

Next, we investigated LTZ influence on cerebellar PF-LTD and PF-LTP, which are two plastic mechanisms thought to be responsible for encoding the VOR gain increase and decrease adaptation, respectively (Broussard et al. 2011; Hansel et al. 2001; Kano et al. 2008; Titley et al. 2010).

E2 synthesis inhibition prevents PF-LTP To induce PFLTP we stimulated the parallel fibers in current-clamp mode at $1 \mathrm{~Hz}$ for $5 \mathrm{~min}$ (Coesmans et al. 2004; Lev-Ram et al. 2002). After tetanization, in the control condition, the EPSC amplitudes were significantly increased to $154 \pm 7.9 \%$ of baseline (Fig. $4 \mathrm{a} ; n=8 ; \mathrm{t}=40-45 \mathrm{~min}$ after; $p<0.001$, two-tailed paired $t$ test) while in LTZ, we detected a $97.6 \pm 7.4 \%$ reduction of baseline that was not significant (Fig. 4a; $n=8 ; t=40-45 \mathrm{~min}$ after; $p=0.16$, two-tailed paired $t$ test). As expected, we found a large significant difference when we compared the EPSC amplitudes in control and LTZ $\left[F_{\mathrm{LTZ} \times \text { control }}(1\right.$, $14)=15.81, p=0.0016 ; F_{\text {induction }}(1,14)=51.2$, $p=0.0001 ; F_{\text {interaction }}(1,14)=80.3, p<0.0001$; two-way repeated measures ANOVA]. Moreover, in LTZ, LTP was induced only in one case and in another case, LTD was induced instead of LTP, suggesting that the main effect of E2 synthesis inhibition is to prevent long-term synaptic changes (Fig. 4c; LTP occurrence: LTZ $\times$ control, $p=0.01$; LTD occurrence: LTZ $\times$ control, $p=1$; no effect occurrence: LTZ $\times$ control, $p=0.04$; two-tailed Fisher's exact test).

Likewise during PF-LTP the PPR (Fig. 4b) changed in neither the control condition (pre-value $1.84 \pm 0.25$, postvalue $1.80 \pm 0.27, p=0.75$; two-tailed paired $t$ test) nor the LTZ condition (pre-value: $1.86 \pm 0.09$, post-value: $1.90 \pm 0.21, p=0.52$; two-tailed paired $t$ test) indicating a post-synaptic PF-LTP expression (Coesmans et al. 2004; Lev-Ram et al. 2002) with no effect on the Pr.

Together, these data suggest that E2 is involved in cerebellar LTP induction likely acting post-synaptically.

E2 synthesis inhibition does not affect PF-LTD After LTD induction in current-clamp mode by paired PF and climbing fiber (CF) stimulation at $1 \mathrm{~Hz}$ for $5 \mathrm{~min}$ (Coesmans et al. 2004), the EPSC amplitudes, recorded in voltage-clamp mode, decreased significantly to $77 \pm 7.3 \%$ of baseline (Fig. 4d; $n=8 ; t=40-45$ min after; $p<0.001$, two-tailed paired $t$ test). The EPSC amplitudes were also reduced by the paired stimulation in the presence of LTZ to $81 \pm 6.5 \%$ of baseline (Fig. 4d; $n=8 ; t=35-40$ min after; $p<0.05$, twotailed paired $t$ test). However, when we compared the EPSC reductions in LTZ and control conditions we found no significant differences $\left[F_{\mathrm{LTZ} \times \text { control }}(1,14)=0.71, p=0.36\right.$; $F_{\text {induction }}(1,14)=31.1, p=0.0001 ; F_{\text {interaction }}(1,14)=3.2$, $p=0.05$; two-way repeated measure ANOVA]. In addition, LTZ did not even change the frequency of LTD occurrence (Fig. 4f; LTZ $\times$ control, $p=0.5$; two-tailed Fisher's exact test). These data suggest that neurosteroid E2 is not involved in cerebellar LTD induction.

Moreover, since long-term plasticity can involve different synaptic changes such as Pr and post-synaptic modifications, we measured the paired pulse ratio (PPR). After tetanization, the PPR (Fig. 4e) did not change in either control condition (pre-value $1.74 \pm 0.19$, post-value $1.85 \pm 0.26, p=0.21$; twotailed paired $t$ test) or LTZ (pre-value $1.73 \pm 0.37$ post-value $1.81 \pm 0.39, p=0.27$; two-tailed paired $t$ test), consistent with a post-synaptic PF-LTD expression (Coesmans et al. 2004; Wang and Linden 2000) and no effect of LTZ on Pr.

\section{Discussion}

The experiments presented in this study show that in male rats the neurosteroid E2 has a relevant impact on the expression of VOR adaptation and regulates cerebellar synaptic plasticity affecting PF-LTP. These findings are consistent with the idea that the cerebellum of young adult rats might be a possible structure where the central nervous system can rapidly modulate E2 bioavailability to influence neuronal activity.

\section{Neurosteroid E2 is necessary for VOR adaptation}

Evidence that E2 impacts the expression of VOR adaptation is that acute block of E2 synthesis by the aromatase inhibitor LTZ prevented VOR gain changes in gain-down 
A
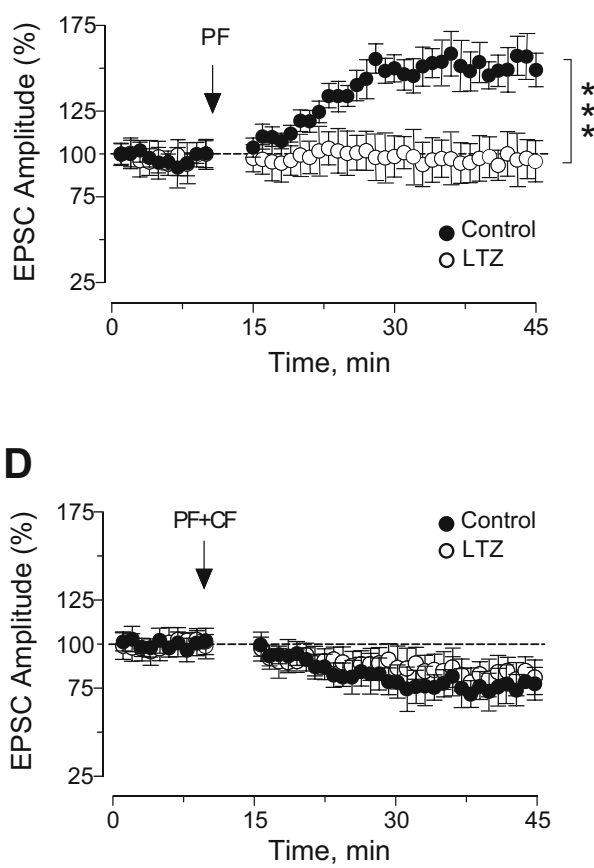

B

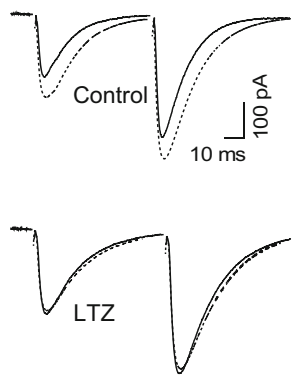

$\mathbf{E}$

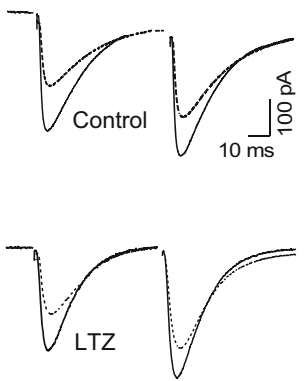

C

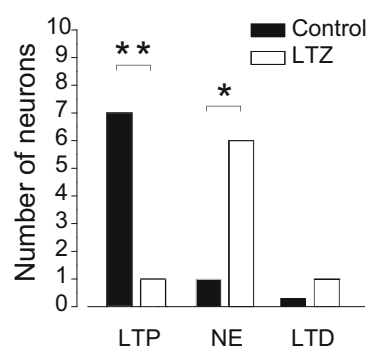

$\mathbf{F}$

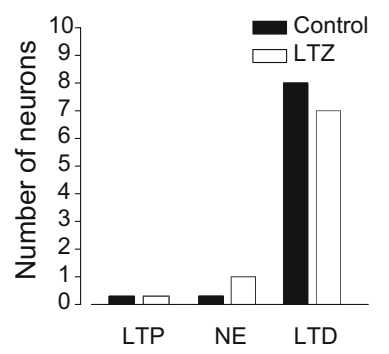

Fig. 4 LTZ blocks LTP but not LTD. a LTP induction by PF stimulation at $1 \mathrm{~Hz}$ increased the EPSCs amplitude of 54\% of control. The induction was blocked when $1 \mathrm{nM}$ LTZ was perfused in the recording chamber (two-way repeated measures ANOVA). b Examples of superimposed EPSC traces before tetanization (line) and $30 \mathrm{~min}$ after $1 \mathrm{~Hz}-\mathrm{PF}$ stimulation (dashed line), recorded before (control, top) and after LTZ (bottom). c Frequency of LTP, no effect (NE) and LTD induced by PF stimulation in control condition and when LTZ was perfused in the recording chamber. The main effect of LTZ was preventing long-term synaptic changes (two-tailed Fisher's exact test). d LTD induction by pairing PF-CF stimulation at $1 \mathrm{~Hz}$

conditioning (Figs. 1, 2, 3b) and converted the gain increase into a decrease in gain-up conditioning (Figs. 1, 2, $3 c)$. Furthermore, the change of the VOR adaptive phase was averted after LTZ administration (Fig. 3). We assume that these effects were related to the inhibition of neurosteroid synthesis rather than the inhibition of gonadal E2 synthesis because in male rats the blood levels of E2 are very low and do not influence, per se, neuronal activity $(\mathrm{Gu}$ and Moss 1996; Woolley 2007).

Moreover, the effect of LTZ appears to be due to the impact of E2 synthesis inhibition on the plasticity of the visuo-vestibular network since no changes were found in basal optokinetic and vestibular reflexes after LTZ administration. In support of these data, it has been shown that inhibiting E2 synthesis using a dose of aromatase imidazole derivative inhibitors similar to the one used in this study, can rapidly affect the adaptive behavior of rodents (Alejandre-Gomez et al. 2007; Graham and Milad 2014; Taziaux et al. 2007). In addition, the effect of the reduced the EPSC amplitudes of $24 \%$ of control. The induction was similar when $1 \mathrm{nM} \mathrm{LTZ}$ was perfused in the recording chamber (twoway repeated measures ANOVA). e Examples of superimposed EPSC traces before tetanization (line) and $30 \mathrm{~min}$ after $1 \mathrm{~Hz}-\mathrm{CF}-\mathrm{PF}$ stimulation (dashed line), recorded before (control, top) and after LTZ (bottom). f Frequency of LTP, NE and LTD induced by PF-CF stimulation in control condition and when LTZ was perfused in the recording chamber. No significant differences were detected between control and LTZ (two-tailed Fisher's exact test). Each trace represents an average of 30 traces. All values are mean $\pm \mathrm{SD}$. $* p<0.05$, $* * p<0.01, * * * p<0.005$, vs. control

LTZ timescale observed in our behavioral experiments is supported by pharmacokinetic results showing that in male rat brain tissue, LTZ reaches high concentration in less than $3 \mathrm{~h}$ after a single oral dose $(2-3.6 \mathrm{mg} / \mathrm{kg})$ with apparent terminal half-life around $12 \mathrm{~h}$ and is completely eliminated from the blood within $36 \mathrm{~h}$ (Liu et al. 2000; Wempe et al. 2007). Yet, we exclude the possibility that the VOR could be influenced by the sequences used to administer the vehicle and LTZ (or the reverse treatment) in the two conditioning sessions that were 8 days apart, since the basal and VOR gain-adaptive changes were not significantly different in the first and the second sessions (Table 1).

The results presented here are also consistent with findings showing rapid effects of exogenous E2 as well as the influence of high circulating E2 levels on memory tasks and learning (Andreescu et al. 2007; Leuner et al. 2004). Indeed, in light of the data, the circulating and local E2 might interact to determine behavioral changes via multiple 
modes and time courses. In line with this idea, the effect of LTZ timescale detected on VOR adaptation could indicate a rapid E2 impact on the molecular pathways leading to synaptic plasticity underling motor memory. This is supported by data demonstrating an effect of LTZ on LTP induction preceding structural remodeling at the synaptic level (Vierk et al. 2012, 2014).

\section{Neurosteroid E2 regulates LTP in acute cerebellar slices}

Evidence that neurosteroid E2 regulates cerebellar synaptic plasticity by acting on LTP, is that acute application of the aromatase inhibitor LTZ in cerebellar slices prevents LTP at the PF-PC synapse without significantly affecting LTD (Fig. 4). Furthermore, we did not find any effects of LTZ on intrinsic and synaptic properties of PCs (Table 2; Fig. 4b, e) in all the conditions tested here. Yet, consistent with the notion that this form of PF-LTP is post-synaptically expressed and E2 exerts an effect at the post-synaptic level (Andreescu et al. 2007; Coesmans et al. 2004; LevRam et al. 2002), we did not find any modification in the PPR in either the control condition or LTZ condition during PF-LTP (Fig. 4b, e).

Since we did not detect a significant effect of LTZ on basal activity and knowing that the perfusion of E2 changes the efficacy of glutamatergic transmission (Hedges et al. 2012; Smith 1989), we suppose that the E2 effect on LTP could be due to either a transient or an acute increase of E2 synthesis during PF-LTP induction. These results are supported by previous data showing the rapid effects of inhibition of aromatase on synaptic LTP in other systems. Indeed, in hippocampus and striatum the same LTZ dose we used here prevented or remarkably reduced LTP, while in vestibular nuclei LTP was inverted in LTD without affecting the basal synaptic responses and LTD (Grassi et al. 2009a, b, 2010, 2012, 2013; Pettorossi et al. 2013).

Conversely, in vivo studies performed in adult rats under anesthesia and using a less specific blocker of E2 synthesis (fadrozole) reported an acute effect of E2 synthesis inhibition on basal glutamatergic transmission at PF synapses (Hedges et al. 2012). A possible explanation to these contrasting results might be the use of diverse techniques that could differently affect network activity and presumably E2 synthesis (Charlier et al. 2015).

We suggest that local concentrations of E2 might fluctuate on demand, rapidly modulating neural transmission when necessary. This idea is in line with evidence showing that aromatase activity can be rapidly modified by glutamate agonists (Balthazart et al. 2006) and phosphorylation processes (Charlier et al. 2011) as well as after an increase in presynaptic or post-synaptic $\mathrm{Ca}^{2+}$ levels (Balthazart et al. 2006; Hojo et al. 2008).

\section{Possible E2 functions on PF plasticity in adult cerebellum}

Taken together, our behavioral and electrophysiological findings suggest a cerebellar E2 synthesis in young adult rats that might be adequate to regulate synaptic plasticity at the PF synapse, despite the fact that high aromatase expression has been shown only in developmental cerebellum. Nevertheless, different studies have shown large variability in aromatase levels in some brain regions (Shimotakahara et al. 2004; Tabatadze et al. 2014) and one study has provided indirect evidence of the efficacy of E2 synthesis in the adult cerebellum of rodents (Hedges et al. 2012). In addition, no clear information is available as to the significance of low aromatase expression in regions of the adult brain like the PCs of the cerebellum (Sakamoto et al. 2003). It is accepted that estrogen, when produced in large amounts, as it is in the amygdala, might influence neuronal functions. Our findings, in contrast, are consistent with the idea that even minute and extremely localized estrogen production may be adequate for modulating local plasticity functions as may be the case in cerebellar PF synapse plasticity.

Thus, local E2 could contribute to synaptic plasticity through pathways associated with rapid and moderate increases in calcium concentration that have been shown to be responsible for LTP in PCs (Coesmans et al. 2004; Jorntell and Hansel 2006; Nilsen et al. 2002; Wang et al. 2014). Consistent with this idea, E2 may facilitate LTP induction as well as prevent inappropriate calcium increments that would switch the LTP into LTD (Fig. 4a, c). On the other hand, E2 might have less importance inducing LTD because CF recruitment is able, per se, to cause a large and prolonged calcium increase in PC dendritic spines related to LTD (Coesmans et al. 2004; Tempia et al. 2001; Vogt and Canepari 2010; Wang et al. 2000).

\section{Cerebellar and possible extra-cerebellar plastic sites for VOR adaptation}

Although the plastic sites for VOR adaptation are thought to be in the flocculus (Boyden and Raymond 2003; Broussard et al. 2011), we conducted our study in the vermis due to technical problems of access to the flocculus. We assumed that the results obtained in the vermis can be generalized to other regions of the cerebellar cortex because of the homogeneity of synaptic plasticity in this brain region.

Since the encoding of adaptive VOR decrease is related to LTP at PF-PC synapses (Boyden and Raymond 2003; Broussard et al. 2011; Coesmans et al. 2004; Hansel et al. 2001), it is possible that acute disruption of E2 synthesis impairs the gain decrease because in the cerebellum, as we 


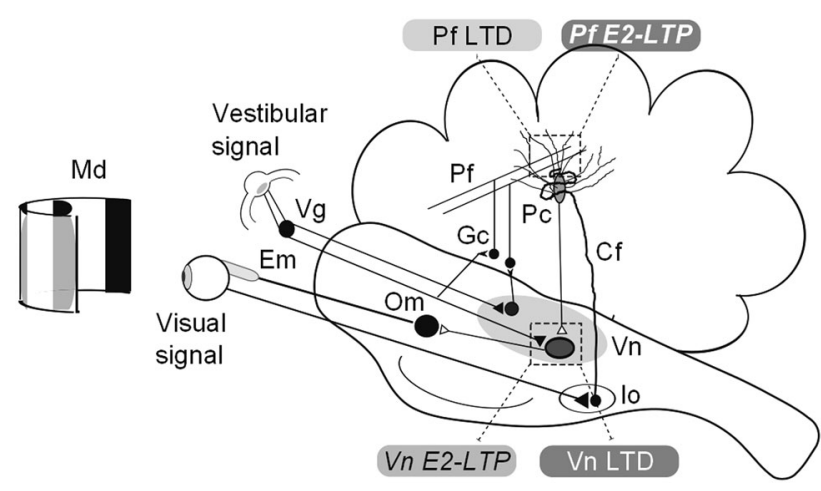

Fig. 5 Cartoon representing the basic visuo-vestibular circuitry and the influence of neurosteroid E2 on the plastic mechanisms that might be responsible for VOR gain adaptation. In the model suggested here, the memory trace for VOR adaptation is stored among cerebellar and vestibular plastic sites, and E2 regulates Pf-LTP and Vn-LTP. In this schema, the gain increase is mediated by Pf-LTD and Vn-E2 LTP (gray shading-black writing) and the gain decrease by Pf-E2 LTP and Vn-LTD (gray shading -white writing). Closed triangle, glutamatergic synapses. Open triangle, GABA or glycinergic synapses. Cf, climbing fiber; Em, extra-ocular muscles; Gc, granule cell layer; Io, inferior olive; Md, moving drum; Om, ocular motoneurons; Pc, Purkinje cell; Pf, parallel fiber; Pf-E2 LTP, E2 dependent LTP at the parallel fiber to Purkinje cell; Pf LTD, LTD at the parallel fiber to Purkinje cell; Pf LTP, LTP at the parallel fiber to Purkinje cell; Vg, vestibular ganglion; Vn, vestibular nuclei; Vn-E2 LTP, E2-dependent LTP in the vestibular nuclei; Vn-LTD, LTD in the vestibular nuclei

demonstrated, local E2 is required to induce LTP at the PF-PCs. On the other hand, the finding that LTZ also impairs the gain increase was unexpected because it should depend on LTD at PF-PC synapses (Boyden and Raymond 2003; Coesmans et al. 2004; Hansel et al. 2006) and this form of plasticity is not affected by E2 (see above electrophysiological results) (Andreescu et al. 2007). To explain why LTZ impairs the adaptive gain increase, we propose a scenario where VOR motor memory is stored among cerebellar and extra-cerebellar vestibular plastic sites (Fig. 5).

We speculate that cerebellar LTP and vestibular LTD might mediate the expression of the VOR adaptive decrease, whereas cerebellar LTD and vestibular LTP might take part in the VOR increase. In this schema, LTZ would prevent the decrease in gain-down conditioning, mainly inhibiting LTP induction in the cerebellum while in gain-up conditioning, the gain increase is reversed into gain decrease causing the inversion of vestibular LTP into LTD (Fig. 5).

Our explanation is supported by evidence based on different approaches showing that the neural networks mediating the VOR contain different potential plastic sites (Blazquez et al. 2004; Boyden et al. 2004; De Zeeuw and Yeo 2005; Hansel et al. 2001; van Alphen and De Zeeuw 2002). It has been also suggested that in long-lasting visuo-vestibular training, motor memory is stored at different cerebellum and vestibular nuclei loci, resulting in memory encoding first in the cerebellum that is then "transferred" to vestibular nuclei (Broussard et al. 2011; Galiana 1986; Kassardjian et al. 2005; Raymond et al. 1996). In addition, a recent model (Menzies et al. 2010) indicates that the "transfer" of motor memory may occur immediately after memory encoding, which is detectable within minutes after the start of the conditioning training. Finally, our previous investigations in vestibular nuclei have demonstrated that locally synthesized E2 is essential in vestibular LTP and more importantly, that LTZ inverts LTP into LTD (Grassi et al. 2009b; Scarduzio et al. 2013).

\section{Conclusion}

In summary, the data presented here show that in male rats the neurosteroid E2 has a relevant impact on the expression of VOR adaptation. Moreover, we demonstrated that neurosteroid E2 regulates cerebellar synaptic plasticity by acting on PF-LTP but not PF-LTD. These findings suggest that very low but extremely localized estrogen production, as could be the case of PCs, might modulate synaptic plasticity. Finally, our results also imply that E2 might regulate VOR adaptation by acting locally on cerebellar and extra-cerebellar synaptic plastic sites.

Acknowledgements We thank Dr. Linda Overstreet-Wadiche and Dr. Jacques Wadiche for advice on electrophysiological procedures and comments throughout this project. This work was supported by the Italian Ministry of Health (Research of Ordinary Projects \# WFR RF-2011-02352379) and by the Cassa di Risparmio di Perugia Foundation.

Open Access This article is distributed under the terms of the Creative Commons Attribution 4.0 International License (http://creativecommons. org/licenses/by/4.0/), which permits unrestricted use, distribution, and reproduction in any medium, provided you give appropriate credit to the original author(s) and the source, provide a link to the Creative Commons license, and indicate if changes were made.

\section{References}

Alejandre-Gomez M, Garcia-Segura LM, Gonzalez-Burgos I (2007) Administration of an inhibitor of estrogen biosynthesis facilitates working memory acquisition in male rats. Neurosci Res 58:272277. doi:10.1016/j.neures.2007.03.011

Andreescu CE et al (2007) Estradiol improves cerebellar memory formation by activating estrogen receptor beta. J Neurosci 27:10832-10839. doi:10.1523/JNEUROSCI.2588-07.2007

Balthazart J, Baillien M, Ball GF (2006) Rapid control of brain aromatase activity by glutamatergic inputs. Endocrinology 147:359-366. doi:10.1210/en.2005-0845

Barmack NH, Nelson BJ (1987) Influence of long-term optokinetic stimulation on eye movements of the rabbit. Brain Res 437:111-120

Baulieu EE (1997) Neurosteroids: Of the nervous system, by the nervous system, for the nervous system. In: Conn PM (ed) 
Recent progress in hormone research, Proceedings of the 1996 conference, vol 52, pp 1-32

Blazquez PM, Hirata Y, Highstein SM (2004) The vestibulo-ocular reflex as a model system for motor learning: what is the role of the cerebellum? Cerebellum 3:188-192. doi:10.1080/ 14734220410018120

Boyden ES, Raymond JL (2003) Active reversal of motor memories reveals rules governing memory encoding. Neuron 39:10311042

Boyden ES, Katoh A, Raymond JL (2004) Cerebellum-dependent learning: the role of multiple plasticity mechanisms. Annu Rev Neurosci 27:581-609. doi:10.1146/annurev.neuro.27.070203. 144238

Boyden ES, Katoh A, Pyle JL, Chatila TA, Tsien RW, Raymond JL (2006) Selective engagement of plasticity mechanisms for motor memory storage. Neuron 51:823-834. doi:10.1016/j.neuron. 2006.08.026

Broussard DM, Titley HK, Antflick J, Hampson DR (2011) Motor learning in the VOR: the cerebellar component. Exp Brain Res 210:451-463. doi:10.1007/s00221-011-2589-Z

Charlier TD, Harada N, Balthazart J, Cornil CA (2011) Human and quail aromatase activity is rapidly and reversibly inhibited by phosphorylating conditions. Endocrinology 152:4199-4210. doi:10.1210/en.2011-0119

Charlier TD, Cornil CA, Patte-Mensah C, Meyer L, Mensah-Nyagan AG, Balthazart J (2015) Local modulation of steroid action: rapid control of enzymatic activity. Front Neurosci 9:83. doi:10. 3389/fnins.2015.00083

Coesmans M, Weber JT, De Zeeuw CI, Hansel C (2004) Bidirectional parallel fiber plasticity in the cerebellum under climbing fiber control. Neuron 44:691-700. doi:10.1016/j.neuron.2004.10.031

Collewijn H, Verhagen AM, Grootendorst AF (1980) Adaptation of the vestibulo-ocular reflex in albino rabbits by selective exposure of the anterior sector of the visual field. Brain Res 192:305-312

Compagnone NA, Mellon SH (2000) Neurosteroids: biosynthesis and function of these novel neuromodulators. Front Neuroendocrinol 21:1-56. doi:10.1006/frne.1999.0188

De Zeeuw CI, Yeo CH (2005) Time and tide in cerebellar memory formation. Curr Opin Neurobiol 15:667-674. doi:10.1016/j. conb.2005.10.008

Farr SA, Flood JF, Scherrer JF, Kaiser FE, Taylor GT, Morley JE (1995) Effect of ovarian steroids on footshock avoidance learning and retention in female mice. Physiol Behav 58:715-723

Foy MR, Xu J, Xie X, Brinton RD, Thompson RF, Berger TW (1999) 17beta-estradiol enhances NMDA receptor-mediated EPSPs and long-term potentiation. J Neurophysiol 81:925-929

Galiana HL (1986) A new approach to understanding adaptive visualvestibular interactions in the central nervous system. J Neurophysiol 55:349-374

Graham BM, Milad MR (2014) Inhibition of estradiol synthesis impairs fear extinction in male rats. Learn Mem 21:347-350. doi:10.1101/lm.034926.114

Grassi S, Frondaroli A, Dieni C, Scarduzio M (2009a) Effects of 17beta-estradiol on synaptic plasticity in the rat medial vestibular nuclei. Acta Otolaryngol 129:390-394. doi:10.1080/ 00016480802566287

Grassi S, Frondaroli A, Dieni C, Scarduzio M, Pettorossi VE (2009b) Long-term potentiation in the rat medial vestibular nuclei depends on locally synthesized 17beta-estradiol. J Neurosci 29:10779-10783. doi:10.1523/JNEUROSCI.1697-09.2009

Grassi S, Frondaroli A, Scarduzio M, Dutia MB, Dieni C, Pettorossi VE (2010) Effects of 17beta-estradiol on glutamate synaptic transmission and neuronal excitability in the rat medial vestibular nuclei. Neuroscience 165:1100-1114. doi:10.1016/j. neuroscience.2009.11.039
Grassi S, Frondaroli A, Scarduzio M, Dieni CV, Brecchia G, Boiti C, Pettorossi VE (2012) Influence of sex and estrous cycle on synaptic responses of the medial vestibular nuclei in rats: role of circulating 17beta-estradiol. Brain Res Bull 87:319-327. doi:10. 1016/j.brainresbull.2011.11.008

Grassi S, Scarduzio M, Panichi R, Dall'Aglio C, Boiti C, Pettorossi VE (2013) Opposite long-term synaptic effects of 17betaestradiol and 5alpha-dihydrotestosterone and localization of their receptors in the medial vestibular nucleus of rats. Brain Res Bull 97:1-7. doi:10.1016/j.brainresbull.2013.05.006

Gu Q, Moss RL (1996) 17 beta-Estradiol potentiates kainate-induced currents via activation of the cAMP cascade. J Neurosci $16: 3620-3629$

Hansel C, Linden DJ, D’Angelo E (2001) Beyond parallel fiber LTD: the diversity of synaptic and non-synaptic plasticity in the cerebellum. Nat Neurosci 4:467-475. doi:10.1038/87419

Hansel C et al (2006) alphaCaMKII Is essential for cerebellar LTD and motor learning. Neuron 51:835-843. doi:10.1016/j.neuron. 2006.08.013

Hazell GG, Yao ST, Roper JA, Prossnitz ER, O'Carroll AM, Lolait SJ (2009) Localisation of GPR30, a novel G protein-coupled oestrogen receptor, suggests multiple functions in rodent brain and peripheral tissues. J Endocrinol 202:223-236. doi:10.1677/ JOE-09-0066

Hedges VL, Ebner TJ, Meisel RL, Mermelstein PG (2012) The cerebellum as a target for estrogen action. Front Neuroendocrinol 33:403-411. doi:10.1016/j.yfrne.2012.08.005

Hojo Y et al (2008) Estrogen synthesis in the brain-role in synaptic plasticity and memory. Mol Cell Endocrinol 290:31-43. doi:10. 1016/j.mce.2008.04.017

Hojo Y et al (2009) Comparison between hippocampus-synthesized and circulation-derived sex steroids in the hippocampus. Endocrinology 150:5106-5112. doi:10.1210/en.2009-0305

Ito M, Shiida T, Yagi S, Yamamoto S (1974) Proceedings: Control mechanism of vestibular oculomotor reflex by cerebellar flocculus. Nihon Seirigaku Zasshi 36:270

Jorntell H, Hansel C (2006) Synaptic memories upside down: bidirectional plasticity at cerebellar parallel fiber-Purkinje cell synapses. Neuron 52:227-238. doi:10.1016/j.neuron.2006.09. 032

Kano M, Hashimoto K, Tabata T (2008) Type-1 metabotropic glutamate receptor in cerebellar Purkinje cells: a key molecule responsible for long-term depression, endocannabinoid signalling and synapse elimination. Philos Trans R Soc Lond B Biol Sci 363:2173-2186. doi:10.1098/rstb.2008.2270

Kassardjian CD, Tan YF, Chung JY, Heskin R, Peterson MJ, Broussard DM (2005) The site of a motor memory shifts with consolidation. J Neurosci 25:7979-7985. doi:10.1523/JNEUR OSCI.2215-05.2005

Kimoto T et al (2001) Neurosteroid synthesis by cytochrome p450containing systems localized in the rat brain hippocampal neurons: $N$-methyl-D-aspartate and calcium-dependent synthesis. Endocrinology 142:3578-3589. doi:10.1210/endo.142.8.8327

Kimpo RR, Boyden ES, Katoh A, Ke MC, Raymond JL (2005) Distinct patterns of stimulus generalization of increases and decreases in VOR gain. J Neurophysiol 94:3092-3100. doi:10. 1152/jn.00048.2005

Leuner B, Mendolia-Loffredo S, Shors TJ (2004) High levels of estrogen enhance associative memory formation in ovariectomized females. Psychoneuroendocrinology 29:883-890. doi:10.1016/j.psyneuen.2003.08.001

Lev-Ram V, Wong ST, Storm DR, Tsien RY (2002) A new form of cerebellar long-term potentiation is postsynaptic and depends on nitric oxide but not cAMP. Proc Natl Acad Sci USA 99:8389_ 8393. doi: $10.1073 /$ pnas. 122206399 
Lisberger SG, Miles FA (1980) Role of primate medial vestibular nucleus in long-term adaptive plasticity of vestibuloocular reflex. J Neurophysiol 43:1725-1745

Liu XD, Xie L, Zhong Y, Li CX (2000) Gender difference in letrozole pharmacokinetics in rats. Acta Pharmacol Sin 21:680-684

McEwen BS, Alves SE (1999) Estrogen actions in the central nervous system. Endocr Rev 20:279-307. doi:10.1210/edrv.20.3.0365

Menzies JR, Porrill J, Dutia M, Dean P (2010) Synaptic plasticity in medial vestibular nucleus neurons: comparison with computational requirements of VOR adaptation. PLoS One. doi:10.1371/ journal.pone. 0013182

Miles FA, Eighmy BB (1980) Long-term adaptive changes in primate vestibuloocular reflex. I. Behavioral observations. J Neurophysiol 43:1406-1425

Moradpour F, Naghdi N, Fathollahi Y (2006) Anastrozole improved testosterone-induced impairment acquisition of spatial learning and memory in the hippocampal CA1 region in adult male rats. Behav Brain Res 175:223-232. doi:10.1016/j.bbr.2006.08.037

Morissette $\mathrm{M}$ et al (2008) Contribution of estrogen receptors alpha and beta to the effects of estradiol in the brain. J Steroid Biochem Mol Biol 108:327-338. doi:10.1016/j.jsbmb.2007.09. 011

Mukai H et al (2007) Rapid modulation of long-term depression and spinogenesis via synaptic estrogen receptors in hippocampal principal neurons. J Neurochem 100:950-967. doi:10.1111/j. 1471-4159.2006.04264.x

Mukai $\mathrm{H}$ et al (2010) Modulation of synaptic plasticity by brain estrogen in the hippocampus. Biochim Biophys Acta 1800:1030 1044. doi:10.1016/j.bbagen.2009.11.002

Nilsen J, Chen S, Brinton RD (2002) Dual action of estrogen on glutamate-induced calcium signaling: mechanisms requiring interaction between estrogen receptors and src/mitogen activated protein kinase pathway. Brain Res 930:216-234

Pettorossi VE, Frondaroli A, Grassi S (2011) Cyclic estrogenic fluctuation influences synaptic transmission of the medial vestibular nuclei in female rats. Acta Otolaryngol 131:434 439. doi:10.3109/00016489.2010.536992

Pettorossi VE, Di Mauro M, Scarduzio M, Panichi R, Tozzi A, Calabresi P, Grassi S (2013) Modulatory role of androgenic and estrogenic neurosteroids in determining the direction of synaptic plasticity in the CA1 hippocampal region of male rats. Physiol Rep 1:e00185. doi:10.1002/phy2.185

Raymond JL, Lisberger SG, Mauk MD (1996) The cerebellum: a neuronal learning machine? Science 272:1126-1131

Raz L, Khan MM, Mahesh VB, Vadlamudi RK, Brann DW (2008) Rapid estrogen signaling in the brain. Neurosignals 16:140-153. doi:10.1159/000111559

Sakamoto H, Mezaki Y, Shikimi H, Ukena K, Tsutsui K (2003) Dendritic growth and spine formation in response to estrogen in the developing Purkinje cell. Endocrinology 144:4466-4477. doi:10.1210/en.2003-0307

Scarduzio M, Panichi R, Pettorossi VE, Grassi S (2013) Synaptic long-term potentiation and depression in the rat medial vestibular nuclei depend on neural activation of estrogenic and androgenic signals. PLoS One 8:e80792. doi:10.1371/journal. pone. 0080792

Shimotakahara S, Hojo S, Furihata K, Tashiro M (2004) Computational and NMR analyses for the identification of bound water molecules in ribonuclease T1. Anal Sci 20:1471-1474

Sirkin DW, Hess BJ, Precht W (1985) Optokinetic nystagmus in albino rats depends on stimulus pattern. Exp Brain Res 61:218221
Smith SS (1989) Estrogen administration increases neuronal responses to excitatory amino acids as a long-term effect. Brain Res 503:354-357

Tabatadze N, Sato SM, Woolley CS (2014) Quantitative analysis of long-form aromatase mRNA in the male and female rat brain. PLoS One 9:e100628. doi:10.1371/journal.pone.0100628

Taziaux M, Keller M, Bakker J, Balthazart J (2007) Sexual behavior activity tracks rapid changes in brain estrogen concentrations. J Neurosci 27:6563-6572. doi:10.1523/JNEUROSCI.1797-07. 2007

Tempia F, Alojado ME, Strata P, Knopfel T (2001) Characterization of the mGluR(1)-mediated electrical and calcium signaling in Purkinje cells of mouse cerebellar slices. J Neurophysiol 86:1389-1397

Titley HK, Heskin-Sweezie R, Broussard DM (2010) The bidirectionality of motor learning in the vestibulo-ocular reflex is a function of cerebellar mGluR1 receptors. J Neurophysiol 104:3657-3666. doi:10.1152/jn.00664.2010

Tsutsui K, Ukena K, Sakamoto H, Okuyama S, Haraguchi S (2011) Biosynthesis, mode of action, and functional significance of neurosteroids in the purkinje cell. Front Endocrinol (Lausanne) 2:61. doi:10.3389/fendo.2011.00061

van Alphen AM, De Zeeuw CI (2002) Cerebellar LTD facilitates but is not essential for long-term adaptation of the vestibulo-ocular reflex. Eur J Neurosci 16:486-490

van Alphen AM, Stahl JS, De Zeeuw CI (2001) The dynamic characteristics of the mouse horizontal vestibulo-ocular and optokinetic response. Brain Res 890:296-305

Vierk R et al (2012) Aromatase inhibition abolishes LTP generation in female but not in male mice. J Neurosci 32:8116-8126. doi:10.1523/JNEUROSCI.5319-11.2012

Vierk R, Brandt N, Rune GM (2014) Hippocampal estradiol synthesis and its significance for hippocampal synaptic stability in male and female animals. Neuroscience 274:24-32. doi:10.1016/j. neuroscience.2014.05.003

Vogt KE, Canepari M (2010) On the induction of postsynaptic granule cell-Purkinje neuron LTP and LTD. Cerebellum 9:284290. doi:10.1007/s12311-010-0174-9

Wang YT, Linden DJ (2000) Expression of cerebellar long-term depression requires postsynaptic clathrin-mediated endocytosis. Neuron 25:635-647

Wang SS, Denk W, Hausser M (2000) Coincidence detection in single dendritic spines mediated by calcium release. Nat Neurosci 3:1266-1273. doi:10.1038/81792

Wang DJ et al (2014) Long-term potentiation at cerebellar parallel fiber-Purkinje cell synapses requires presynaptic and postsynaptic signaling cascades. J Neurosci 34:2355-2364. doi:10.1523/ JNEUROSCI.4064-13.2014

Wempe MF et al (2007) Pharmacokinetics of letrozole in male and female rats: influence of complexation with hydroxybutenyl-beta cyclodextrin. J Pharm Pharmacol 59:795-802. doi:10.1211/jpp. 59.6 .0006

Woolley CS (2007) Acute effects of estrogen on neuronal physiology. Annu Rev Pharmacol Toxicol 47:657-680. doi:10.1146/annurev. pharmtox.47.120505.105219

Woolley CS, McEwen BS (1994) Estradiol regulates hippocampal dendritic spine density via an N-methyl-D-aspartate receptordependent mechanism. J Neurosci 14:7680-7687

Xu X, Zhang Z (2006) Effects of estradiol benzoate on learningmemory behavior and synaptic structure in ovariectomized mice. Life Sci 79:1553-1560. doi:10.1016/j.lfs.2006.04.020 